\title{
A Novel E-DVA Module Synthesis Featuring of Synergy between Driving and Vibration Attenuation
}

\author{
Cheng Gu, ${ }^{1,2}$ Jianfei Zhu, ${ }^{3}$ and Xinbo Chen ${ }^{1,2}$ \\ ${ }^{1}$ College of Automotive Studies, Tongji University, Shanghai 201804, China \\ ${ }^{2}$ Clean Energy Automotive Engineering Center, Tongji University, Shanghai 201804, China \\ ${ }^{3}$ College of Mechanical Engineering, Tongji University, Shanghai 201804, China
}

Correspondence should be addressed to Xinbo Chen; austin_1@aliyun.com

Received 18 April 2016; Accepted 9 June 2016

Academic Editor: Salvatore Strano

Copyright (C) 2016 Cheng Gu et al. This is an open access article distributed under the Creative Commons Attribution License, which permits unrestricted use, distribution, and reproduction in any medium, provided the original work is properly cited.

\begin{abstract}
To attenuate the negative effects brought by heavy unsprung mass of the decentralized driving electric vehicle, a novel e-DVA module featuring of synergy between driving and vibration attenuation is proposed in this paper. It presents the advantages of compact structure and low cost. Structure design proves the feasibility of the e-DVA module. Kinematic analysis of the slidercrank mechanism is carried out to conclude the transmission ratio ripple under road excitation. After parameter matching and optimization of the e-DVA module based on the $\mathrm{H}_{2} / \mathrm{H}_{\infty}$ norm criterions, vertical dynamics analyses in both frequency and time domains are conducted theoretically to prove the performance improvements on the ride comfort and handling stability under the constraint of DVA deflection bound.
\end{abstract}

\section{Introduction}

Regarding fossil fuel saving and environmental protection, some research institutions and corporations have proposed a variety of electric vehicles, the majority of which are based on the conventional internal combustion engine platform [1]. Although it is still in the stage of concept promotion featuring of individual driving wheels, the decentralized driving electric vehicle has shown some advantages. Eliminating the components including differential in the simplified chassis, the space within vehicle body is preserved. In addition, as driving torque on each wheel can be achieved and controlled precisely, it provides new possibilities for the dynamics control strategies [2]. However, fixing the motor in wheel or close to wheel increases the unsprung mass, which deteriorates the performances of ride comfort, handling stability, and safety. As a result, its promotion is hindered [3].

To reduce the unsprung mass, considerable investigations have been done by researchers around the world. Except lightweight designs on material and structure $[4,5]$, positioning the motor on the vehicle body is a common idea to transfer it as a sprung mass [6], as depicted in Figure 1. However, with the introduction of universal joints, the transmission system efficiency is reduced, and the stiffness of the elongated shaft is comparatively small.

In recent years, a lot of studies on active suspension and semiactive suspension are carried out by researchers $[7,8]$. In spite of the improvements on ride comfort and handling stability, high cost actuators and complicated controllers contribute to the failure to be widely promoted. Consequently, designs based on passive suspension are more practicable approaches for vehicles in production at present.

Our research group has been investigating techniques to attenuate the negative effects brought by heavy unsprung mass. Taking the gearbox as a trailing arm, Figure 2(a) illustrates an integral of a driving system and chassis in a top view [9]. Taking the vehicle body as a reference, as the motor swings far away from the wheel under excitation, the vibration amplitude of the motor is much smaller than the wheel. Consequently, the negative influences of unsprung mass are reduced comparing with the architecture of hub motor direct driving, and it improves compactness as the 


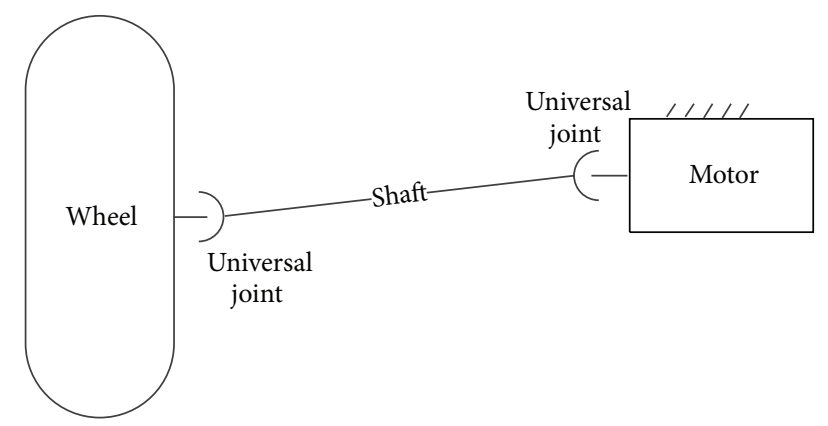

FIGURE 1: Schematic diagram of positioning the motor on the vehicle body.

shaft and universal joints are eliminated. Furthermore, with the introduction of the reducer between the motor and wheel, it fully utilizes the high power density of high speed motor and reduces the cost of motor, especially in the state of low speed and high torque. But the attenuation performance depends on the structure parameter. Study results show that the longer the distance between axles of wheel and motor, the lower the equivalent unsprung mass [10]. And Figure 2(b) shows a prototype application in a decentralized driving electric vehicle.

Dynamic vibration absorber (DVA) has been widely applied to attenuate the resonance vibration of primary system in mechanical systems [11]. But in vehicle suspensions, both the introduction of an auxiliary mass and the space restriction near the wheel limit its application [12, 13].

Taking the driving motor itself as a DVA, a novel electric wheel concept named e-DVA module throughout the paper based on slider-crank mechanism is proposed in Section 2, featuring of the synergy between electric driving and dynamic vibration absorbing. In Section 3, kinematics analyses of the slider-crank mechanism composed by two gear-pole composite mechanism are carried out, and the wheel speed ripple is concluded. In Section 4, under a random excitation, the parameters of the e-DVA module are matched and optimized based on the $\mathrm{H}_{2}$ norms of body acceleration, suspension deflection, and tire dynamic load under the $H_{\infty}$ norm constraint of DVA motion bound. At last, comparing with the in-wheel motored electric wheel, the vertical dynamics analyses are carried out in both frequency and time domains to verify the improvements on ride comfort and handling stability.

\section{E-DVA Module Architecture}

With the advantages of integration and adaptation, modular design is very convenient for mass production and recycling, which shortens the period of investigation and manufacturing [14]. Michelin and Siemens corporations, respectively, proposed the electric wheel modules integrating the hub motor, active suspension, and brake system in wheels $[15,16]$. However, both wheel modules are of complicated structures and high cost for direct driving of hub motor and introducing active suspension.

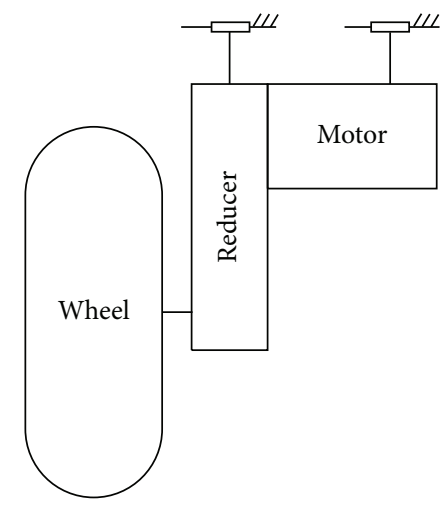

(a) Configuration of the integral

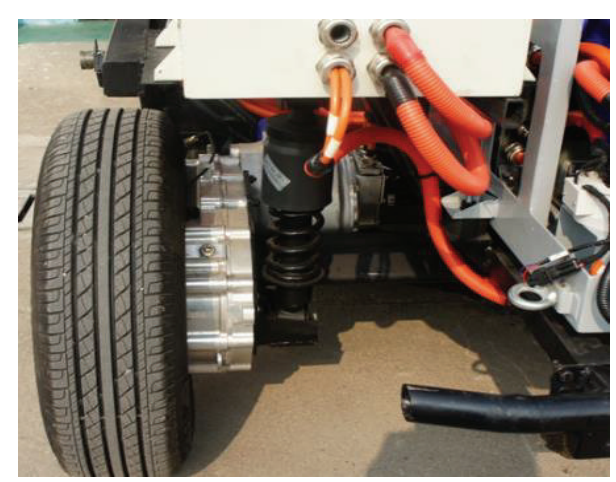

(b) Prototype manufacturing

FIGURE 2: Integral of electric drive and single trailing arm suspension.

A novel e-DVA module architecture is proposed in this section, and a simplified schematic diagram based on slidercrank mechanism is demonstrated in Figure 3. It is mainly composed of the tire, rim, bearing, hub, braking disc, caliper, gearbox, and motor. The output shaft of the motor $M$ is connected with gear $A$ by spline, and the driving shaft is connected with gear $D$ by spline. Gear $A$ is engaged with gear $B$, while gear $C$ is engaged with gear $D$, and gear $B$ shares the same shaft with gear $C$. The two ends of link $a$ are connected with gear $A$ and gear $B$, respectively, while the two ends of link $b$ are connected with gear $C$ and gear $D$. The upper end of the spring is fixed with the motor $M$, and the lower end of the spring is fixed with the knuckle. The motor $M$, the link $a$, the link $b$, the spring, and damper construct a slider-crank mechanism, in which the motor $M$ acts as the slider. Taking the knuckle as a reference, under the excitation of uneven road, the motor $M$ would move up and down along the common axle of the spring and damper, while both link $a$ and link $b$ conduct planer motions, guaranteeing the engagements of the gears in motion.

Detailed structure design is demonstrated in Figure 4. To avoid interference with other components, the motor is constrained moving vertically along the diameter of the rim to fully utilize the space in wheel. For compactness, a novel bearing is adopted, whose inner ring is regarded as the knuckle, while the outer ring is fixed with the rim. So that the components including transmission system can be arranged 


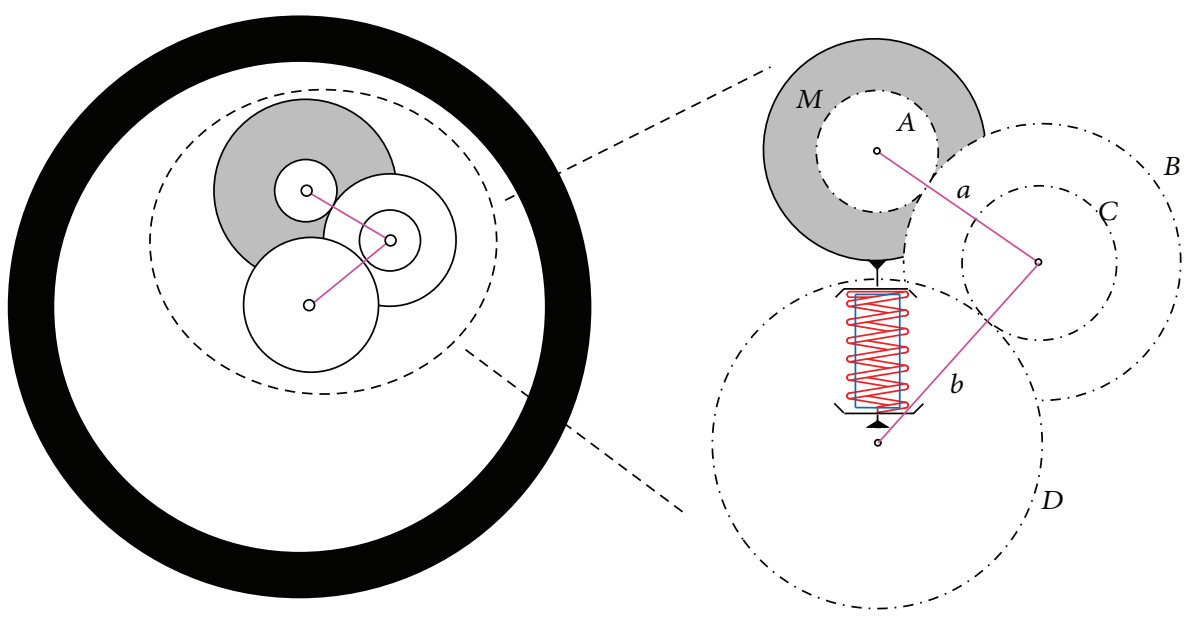

FIGURE 3: E-DVA system concept based on slider-crank mechanism.
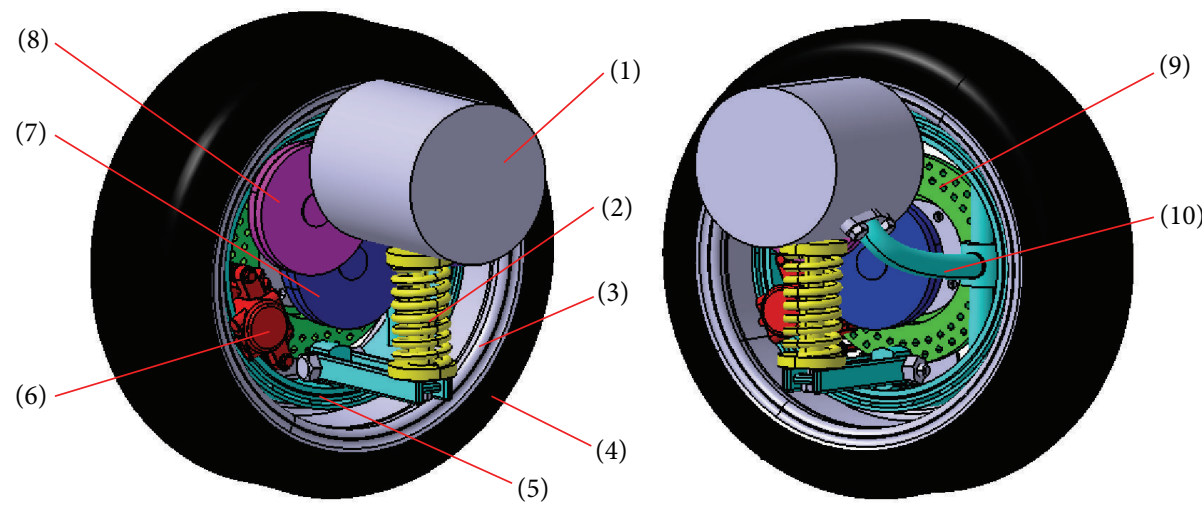
(1) Motor
(2) Spring
(3) Rim
(4) Tire
(5) Bearing
(6) Caliper
(7) Second gearbox
(8) First gearbox
(9) Brake disc
(10) Guide mechanism

Figure 4: E-DVA module architecture.

in wheel, which is originally occupied by the conventional hub bearing and knuckle in sequence. A guide mechanism is introduced to restrict the motor motion.

In the e-DVA module designed for a low speed electric vehicle, a 15 -inch tire is selected. According to the maximum speed requirement of $70 \mathrm{~km} / \mathrm{h}$, the power system parameters are designed and matched as shown in Table 1.

In conclusion, the outstanding feature of the e-DVA system proposed in this paper is the synergy between electric driving and dynamic vibration absorbing. Serving as a DVA, the motor attenuates the resonance vibration of vehicle body without any auxiliary mass. Performing as the links in the slider-crank mechanism, the gearbox decouples the rigid connection between the motor and the wheel and decreases the torque requirements for the motor.

\section{Kinematics Analyses}

In Figure 3, as gear $A$ is engaged with gear $B$ and link $a$ performs the planer motion when the e-DVA module is under excitation, an equivalent planetary gear mechanism composed of gear $A$, gear $B$, and link $a$ is analyzed in kinematics in this section. Supposing that the rotational speeds of gear $A$, gear $B$, and link $a$ are $\omega_{A}, \omega_{B}$, and $\omega_{a}$, respectively, and the teeth of gear $A$ and gear $B$ are $z_{A}$ and $z_{B}$, then the rotational speed relationship shown below is satisfied:

$$
\frac{\omega_{A}-\omega_{a}}{\omega_{B}-\omega_{a}}=-\frac{z_{B}}{z_{A}} .
$$

In a similar way, for the planetary gear mechanism composed of gear $C$, gear $D$, and link $b$, supposing that the 
TABle 1: Power system parameters.

\begin{tabular}{|c|c|c|c|c|c|c|}
\hline & Parameter & Symbol & Value & Parameter & Symbol & Value \\
\hline \multirow{4}{*}{ Motor } & Mass $/ \mathrm{kg}$ & $m_{3}$ & 30 & Radius $/ \mathrm{mm}$ & $r_{0}$ & 95 \\
\hline & Peak speed $/\left(\mathrm{r} \cdot \mathrm{min}^{-1}\right)$ & $n_{p}$ & 5300 & Rated speed $/\left(r \cdot \mathrm{min}^{-1}\right)$ & $n_{r}$ & 3000 \\
\hline & Peak power/kW & $P_{p}$ & 12 & Rated power/kW & $P_{r}$ & 6 \\
\hline & Peak torque/(N.m) & $T_{p}$ & 72 & Rated torque/(N.m) & $T_{r}$ & 20 \\
\hline \multirow[t]{5}{*}{ Wheel } & Mass/kg & $m_{1}$ & 25 & Radius/mm & $r_{w}$ & 190.5 \\
\hline & & First stage & & & cond stag & \\
\hline & Transmission ratio & $i_{1}$ & 3.421 & Transmission ratio & $i_{2}$ & 2.478 \\
\hline & Teeth & $z_{A} / z_{B}$ & $19 / 65$ & Teeth & $z_{C} / z_{D}$ & $23 / 57$ \\
\hline & Smaller gear radius $/ \mathrm{mm}$ & $r_{A}$ & 16 & Smaller gear radius $/ \mathrm{mm}$ & $r_{C}$ & 20 \\
\hline \multirow[t]{4}{*}{ Reducer } & Larger gear radius/mm & $r_{B}$ & 52 & Larger gear radius/mm & $r_{D}$ & 63 \\
\hline & Center distance/mm & $L_{1}$ & 68 & Center distance/mm & $L_{2}$ & 83 \\
\hline & Smaller gear mass $/ \mathrm{kg}$ & $m_{A}$ & 1.7 & Smaller gear mass $/ \mathrm{kg}$ & $m_{C}$ & 2.1 \\
\hline & Larger gear mass $/ \mathrm{kg}$ & $m_{B}$ & 4.3 & Larger gear mass $/ \mathrm{kg}$ & $m_{D}$ & 4.2 \\
\hline
\end{tabular}

rotational speeds of gear $C$, gear $D$, and link $b$ are $\omega_{C}, \omega_{D}$, and $\omega_{b}$, respectively, and the teeth of gear $C$ and gear $D$ are $z_{C}$ and $z_{D}$, then the following equation illustrates the speed relationship:

$$
\frac{\omega_{C}-\omega_{b}}{\omega_{D}-\omega_{b}}=-\frac{z_{D}}{z_{C}}
$$

Gear $B$ and gear $C$ share the same shaft by splines, which means $\omega_{B}=\omega_{C}$.

After computation, the speed of gear $D$ is obtained as follows:

$$
\begin{aligned}
\omega_{D} & =-\frac{z_{C}}{z_{D}}\left(-\frac{z_{A}}{z_{B}} \omega_{A}+\frac{z_{A}+z_{B}}{z_{B}} \omega_{a}\right)+\frac{z_{C}+z_{D}}{z_{D}} \omega_{b} \\
& =\frac{z_{A} \cdot z_{C}}{z_{B} \cdot z_{D}} \omega_{A}-\frac{z_{C}}{z_{D}} \cdot \frac{z_{A}+z_{B}}{z_{B}} \omega_{a}+\frac{z_{C}+z_{D}}{z_{D}} \omega_{b} .
\end{aligned}
$$

It is concluded from the equation above that, as the motor moves along the common axle of spring and damper with respect to knuckle, the transmission ratio is not stable under uneven road excitation. The speed ripple of the wheel can be depicted as follows, which is related to the rotational speeds of link $a$ and link $b$ :

$$
-\frac{z_{C}}{z_{D}} \cdot \frac{z_{A}+z_{B}}{z_{B}} \omega_{a}+\frac{z_{C}+z_{D}}{z_{D}} \omega_{b} .
$$

The influence of wheel speed ripple would be analyzed in Section 4 combining with a specific example.

\section{Vertical Dynamics Analyses}

4.1. Equivalent Mass of Gear System. In this paper, the gear system is introduced to reduce the torque requirement of the motor, and the motor cost is reduced meanwhile. To establish the model precisely, the gear system should be taken into consideration. The influence of the gear system mass on the vertical dynamics is supposed to be analyzed by distributing the gear mass to the motor and wheel, respectively. According to the parallel axis theorem, the first stage reducer is transferred to the motor mass when the moments of inertial are kept equivalent:

$$
\frac{1}{2} m_{3}^{*} r_{0}^{2}=\frac{1}{2} m_{3} r_{0}^{2}+\frac{1}{2} m_{A} r_{A}^{2}+\frac{1}{2} m_{B} r_{B}^{2}+m_{B} L_{1}^{2}
$$

in which $m_{3}^{*}$ is the equivalent DVA mass.

In the same way, the second stage reducer is transferred to the wheel mass:

$$
\frac{1}{2} m_{1}^{*} r_{w}^{2}=\frac{1}{2} m_{1} r_{w}^{2}+\frac{1}{2} m_{C} r_{C}^{2}+\frac{1}{2} m_{D} r_{D}^{2}+m_{D} L_{2}^{2}
$$

in which $m_{1}^{*}$ is the equivalent wheel mass.

It is concluded that, with the introduction of the gear system, the equivalent DVA mass and wheel mass are increased a little, while the unsprung mass is decreased significantly without in-wheel motor.

4.2. Model Establishment. According to the e-DVA module architecture, a quarter vehicle model of three-degree-offreedom (DOF) is established in Figure 5, where $m_{1}^{*}$ is the equivalent wheel mass (the unsprung mass), $m_{2}$ is the sprung mass, and $m_{3}^{*}$ is the equivalent DVA mass; $k_{1}$ is the tire stiffness, $k_{2}$ is the suspension stiffness, and $k_{3}$ is the DVA stiffness; $c_{2}$ and $c_{3}$ represent the suspension damping and DVA damping, respectively, and the tire damping is small enough to be neglected; $q, z_{1}, z_{2}$, and $z_{3}$ are the vertical displacements of the road, wheel, vehicle body, and DVA, respectively.

According to Newton's second law, the dynamic differential equation is established, as shown in

$$
\mathbf{M}\left[\begin{array}{c}
\ddot{z}_{1} \\
\ddot{z}_{2} \\
\ddot{z}_{3}
\end{array}\right]+\mathbf{C}\left[\begin{array}{l}
\dot{z}_{1} \\
\dot{z}_{2} \\
\dot{z}_{3}
\end{array}\right]+\mathbf{K}\left[\begin{array}{c}
z_{1} \\
z_{2} \\
z_{3}
\end{array}\right]=\mathbf{K}_{1} q
$$




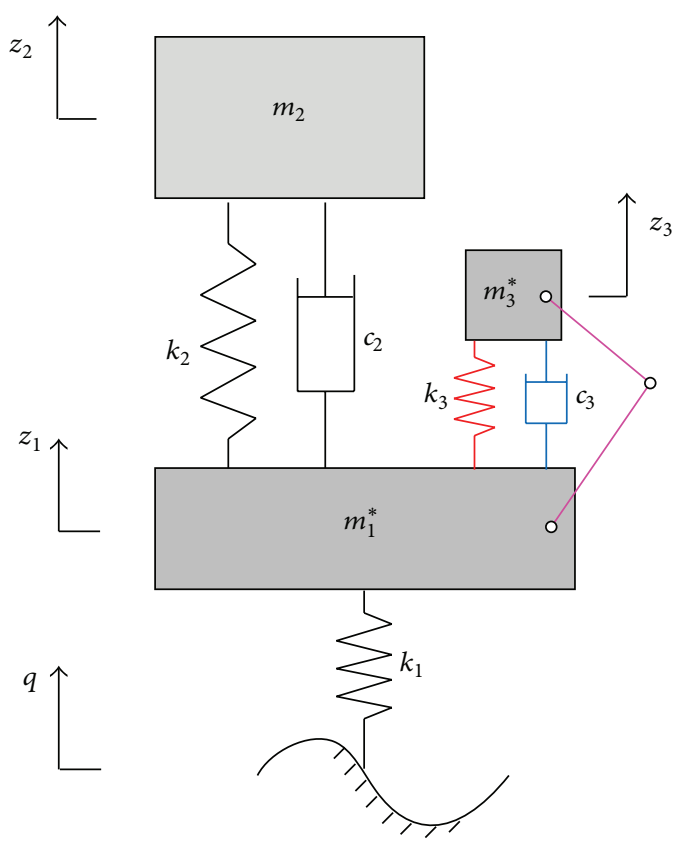

FIgURE 5: A quarter vehicle model of three DOF.

where

the mass matrix is as follows:

$$
\mathbf{M}=\left[\begin{array}{ccc}
m_{1}^{*} & 0 & 0 \\
0 & m_{2} & 0 \\
0 & 0 & m_{3}^{*}
\end{array}\right]
$$

damping matrix is as follows:

$$
\mathbf{C}=\left[\begin{array}{ccc}
c_{2}+c_{3} & -c_{2} & -c_{3} \\
-c_{2} & c_{2} & 0 \\
-c_{3} & 0 & c_{3}
\end{array}\right]
$$

stiffness matrix is as follows:

$$
\mathbf{K}=\left[\begin{array}{ccc}
k_{1}+k_{2}+k_{3} & -k_{2} & -k_{3} \\
-k_{2} & k_{2} & 0 \\
-k_{3} & 0 & k_{3}
\end{array}\right]
$$

The system generalized force is the road excitation $\mathbf{K}_{1} q$, in which

$$
\mathbf{K}_{1}=\left[\begin{array}{c}
k_{1} \\
0 \\
0
\end{array}\right]
$$

Performing Laplace transformation on (7), the transfer functions of the output displacements with road vertical displacement $q$ are concluded, as depicted in

$$
\mathbf{H}=\left[\begin{array}{c}
\frac{z_{1}}{q} \\
\frac{z_{2}}{q} \\
\frac{z_{3}}{q}
\end{array}\right]=\frac{\mathbf{K}_{1}}{\mathbf{K}+\mathbf{C s}+\mathbf{M} s^{2}} .
$$

As a result, to reflect the ride comfort, the transfer function of body acceleration $\ddot{z}_{2}$ with the input vertical speed $\dot{q}$ is shown in

$$
|\mathbf{H}|_{\ddot{z}_{2} \sim \dot{q}}=\left|\frac{\ddot{z}_{2}}{\dot{q}}\right|=s \cdot|\mathbf{H}(2,1)| .
$$

The transfer function of the wheel dynamic load $F_{d}$ with the input vertical speed $\dot{q}$ is depicted as follows, which demonstrates the handling stability:

$$
|\mathbf{H}|_{F_{d} \sim \dot{q}}=\left|\frac{z_{1}-q}{\dot{q}} k_{1}\right|=\frac{1}{s} \cdot\left|[\mathbf{H}(1,1)-1] k_{1}\right| .
$$

The suspension deflection $z_{2}-z_{1}$ and DVA deflection $z_{3}-z_{1}$ should be in their motion bounds, and the transfer functions are illustrated similarly in

$$
\begin{aligned}
& |\mathbf{H}|_{\left(z_{2}-z_{1}\right) \sim \dot{q}}=\left|\frac{z_{2}-z_{1}}{\dot{q}}\right|=\frac{1}{s} \cdot|[\mathbf{H}(2,1)-\mathbf{H}(1,1)]|, \\
& |\mathbf{H}|_{\left(z_{3}-z_{1}\right) \sim \dot{q}}=\left|\frac{z_{3}-z_{1}}{\dot{q}}\right|=\frac{1}{s} \cdot|[\mathbf{H}(3,1)-\mathbf{H}(1,1)]| .
\end{aligned}
$$

4.3. Parameter Matching of E-DVA Module Based on $\mathrm{H}_{2} / \mathrm{H}_{\infty}$ Norms

4.3.1. Optimization Objective Based on $\mathrm{H}_{2} / \mathrm{H}_{\infty}$ Norms. Regarding the parameter optimization of a single freedom DVA with damper, extensive researches have been conducted [17]. The researches aimed at determining dynamic vibration system parameters including the tuning frequency and damping ratio to attenuate the main system vibration. However, in the vehicle suspension design, besides the ride comfort, objectives including handling stability and motion interference should also be considered. Furthermore, regarding an actual system, the driving motor parameters are previously determined by the power requirement of the vehicle. This paper aims at improving the ride comfort and handling stability through optimizing the architecture of the power system without influencing the power performance. Restricted by the motor structure characteristic and manufacturing, the power performance is probably influenced if the motor mass is varied. As a result, although it is feasible to include the motor mass in the optimization, we take the motor mass as a hard constraint. In conclusion, the optimized parameters involve the DVA stiffness and damping under multiobjectives.

As is previously acknowledged, the performances of ride comfort and handling stability are reflected by the vehicle 
body acceleration and tire dynamic load, while suspension deflection should be in the permitted range; otherwise the system dynamics would become extremely nonlinear. As for the e-DVA module in this paper, in addition to the factors considered above, the deflection of the DVA should also be restricted to avoid interference with other components.

However, for a determined primary system and motor mass, adjusting the stiffness and damping of the DVA system has little influence on the peak values at the first-order resonance frequency. Alternately, the objective is to reduce the total vibration energy of the system over all frequencies when the primary system is subjected to random excitation. In this optimization criterion, the RMS values of the frequency response curves are optimized to be minimums. If the input road excitation is independent, the RMS values can be represented by the $\mathrm{H}_{2}$ norms in the physical sense. Consequently, $\mathrm{H}_{2}$ norms of the body acceleration, suspension deflection, and tire dynamic load are taken as the objectives of ride comfort and handling stability.

According to the variation ranges of the above three items, specific weighting coefficients are evaluated to formulate the criterion function of the optimization objective, as depicted in (16) in detail. One has

$$
\min \quad J=\boldsymbol{\alpha} \mathbf{H}_{2}=\left[\begin{array}{lll}
\alpha_{1} & \alpha_{2} & \alpha_{3}
\end{array}\right]\left\|\begin{array}{c}
T_{\ddot{z}_{2} \sim \dot{q}} \\
T_{\left(z_{2}-z_{1}\right) \sim \dot{q}} \\
T_{\left(z_{1}-q\right) k_{1} \sim \dot{q}}
\end{array}\right\|_{2} .
$$

In a similar way, the minimal of the DVA deflection amplitude at the resonance frequency, represented by $H_{\infty}$ norm, is evaluated as the objective of motion interference. $\gamma$ is set as the travel bound, as depicted in

$$
\left\|T_{\left(z_{3}-z_{1}\right) \sim \dot{q}}\right\|_{\infty}<\gamma .
$$

In summary, considering the performance improvements and interference, the objective can be depicted as follows:

$$
\begin{aligned}
\min \quad J & =\boldsymbol{\alpha} \mathbf{H}_{2}+\boldsymbol{\beta} \mathbf{H}_{\infty} \\
& =\left[\begin{array}{lll}
\alpha_{1} & \alpha_{2} & \alpha_{3}
\end{array}\right]\left\|\begin{array}{c}
T_{\ddot{z}_{2} \sim \dot{q}} \\
T_{\left(z_{2}-z_{1}\right) \sim \dot{q}} \\
T_{\left(z_{1}-q\right) k_{1} \sim \dot{q}}
\end{array}\right\|_{2}+\beta\left\|T_{\left(z_{3}-q\right) \sim \dot{q}}\right\|_{\infty} .
\end{aligned}
$$

4.3.2. Parameter Matching of a Specific E-DVA Module. Supposing a vehicle drives on $C$ class road with a speed of $20 \mathrm{~m} / \mathrm{s}$, the power spectrum density (PSD) of road is depicted in

$$
G_{\dot{q}}(f)=4 \pi^{2} G_{q}\left(n_{0}\right) n_{0}^{2} u .
$$

In the previous equation, $n_{0}$ is the referenced space frequency; $n_{0}=0.1 \mathrm{~m}^{-1} ; G q\left(n_{0}\right)$ is the uneven coefficient; $u$ is the vehicle speed. The frequency range of integration is from $0.1 \mathrm{~Hz}$ to $100 \mathrm{~Hz}$.

According to (5)-(6), considering the influence of gear system, the equivalent DVA mass and wheel mass are computed, and simulation parameters of a specific example are listed in Table 2.

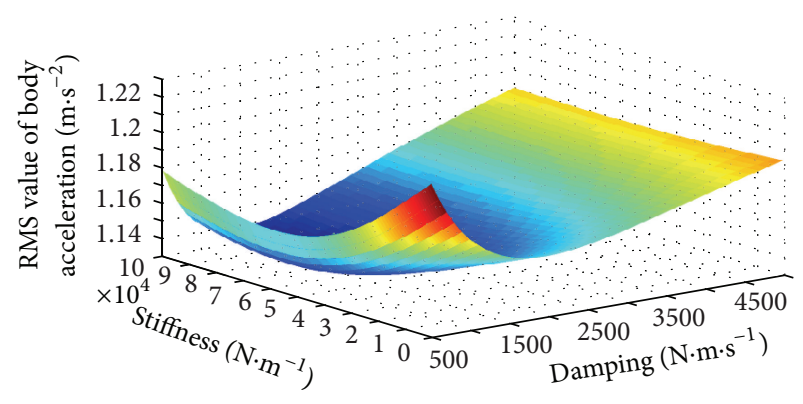

FIGURE 6: RMS map of body acceleration.

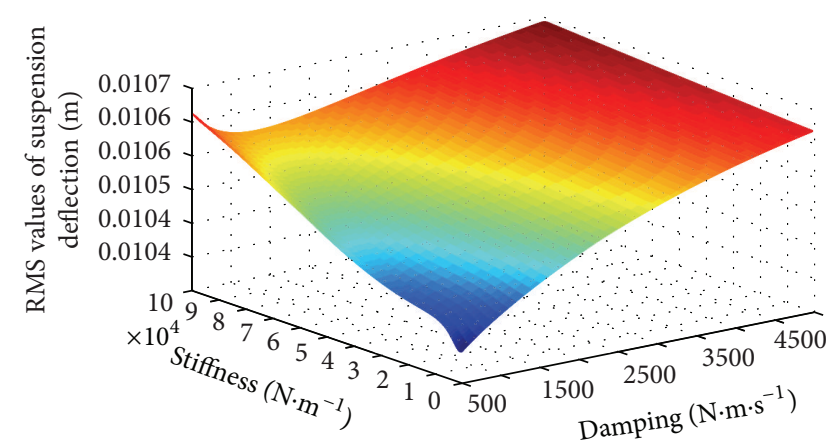

FIgURE 7: RMS map of suspension deflection.

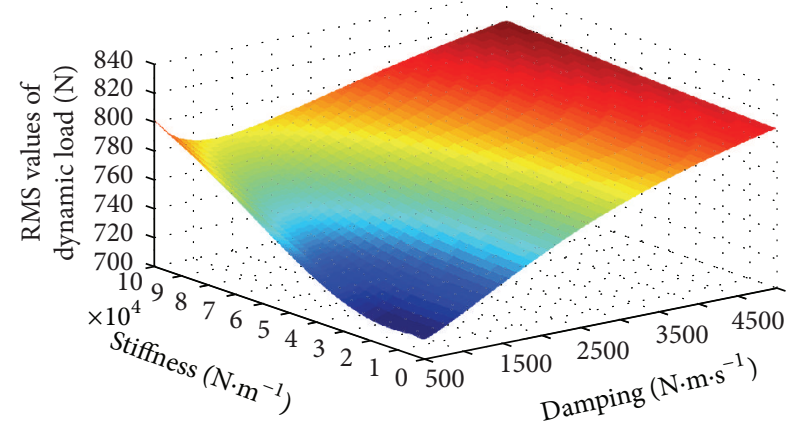

Figure 8: RMS map of dynamic load.

According to the $\mathrm{H}_{2} / \mathrm{H}_{\infty}$ norm criterions, for the e-DVA system with different stiffness and damping matches, Figures 6-8 show the RMS maps of body acceleration, suspension deflection, and tire dynamic load, as well as peak value map of DVA deflection in Figure 9.

The weighting coefficients are determined by the relative importance of the body acceleration, suspension deflection, tire dynamic load, and DVA deflection. However, in the optimization process, it is concluded that the suspension deflection is hardly influenced by the DVA stiffness and damping as its perturbation range is only $0.4 \mathrm{~mm}$, and it locates in a suitable range, as depicted in Figure 7. As a result, the suspension deflection is neglected. In conclusion, as the ride comfort is preferred for passengers, the weighted performances of body acceleration, tire dynamic load, and DVA deflection are set as 6:5:4 in the variation ranges of both DVA stiffness and damping, while the weighting 
TABLE 2: Simulation parameters of $1 / 4$ vehicle model.

\begin{tabular}{lccc}
\hline & Symbol & In-wheel motored system (IMS) & e-DVA system \\
\hline Wheel mass $/ \mathrm{kg}$ & $m_{1}$ & 25 & 26.28 \\
Body mass $/ \mathrm{kg}$ & $m_{2}$ & 400 & 400 \\
Motor mass $/ \mathrm{kg}$ & $m_{3}$ & 30 & 35.74 \\
Tire stiffness $/ \mathrm{N} \cdot \mathrm{m}^{-1}$ & $k_{1}$ & 200000 & 200000 \\
Suspension $\mathrm{stiffness} / \mathrm{N} \cdot \mathrm{m}^{-1}$ & $k_{2}$ & 25000 & 25000 \\
Suspension damping $/(\mathrm{N} \cdot \mathrm{s}) \cdot \mathrm{m}^{-1}$ & $c_{2}$ & 2000 & 2000 \\
\hline
\end{tabular}

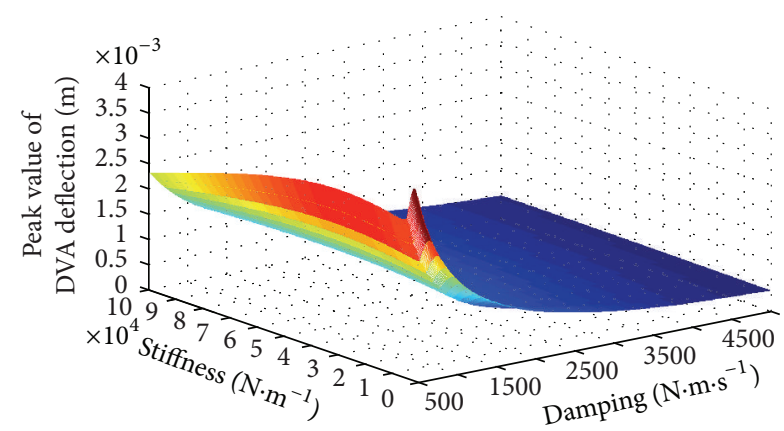

FIGURE 9: Peak value map of DVA deflection.

coefficient of suspension deflection is set as 0 , as shown in the following:

$$
\begin{aligned}
\alpha_{1} \Delta_{\ddot{z}_{2}}: \alpha_{3} \Delta_{k_{1}\left(z_{1}-q\right)}: \beta \Delta_{z_{3}-z_{1}} & =6: 5: 4, \\
\alpha_{2} & =0 .
\end{aligned}
$$

From Figures 7-9, the variation ranges of suspension performances are concluded as follows:

$$
\begin{aligned}
\Delta_{\ddot{z}_{2}} & =0.15, \\
\Delta_{k_{1}\left(z_{1}-q\right)} & =150, \\
\Delta_{z_{3}-z_{1}} & =0.004 .
\end{aligned}
$$

Consequently, the weighting matrices $\boldsymbol{\alpha}$ and $\boldsymbol{\beta}$ are determined:

$$
\begin{aligned}
& \boldsymbol{\alpha}=\left[\begin{array}{lll}
1200 & 0 & 1
\end{array}\right], \\
& \boldsymbol{\beta}=30000
\end{aligned}
$$

The suboptimal solution of (16) can be concluded, in which the DVA stiffness $k_{3}$ is $42000 \mathrm{~N} / \mathrm{m}$ and damping $c_{3}$ is $1200 \mathrm{~N} \cdot \mathrm{s} / \mathrm{m}$.

\subsection{Simulation in Frequency and Time Domain}

4.4.1. Simulation in Frequency Domain. With the optimized parameters above, according to the transfer functions in (13)-(15), the power spectral density (PSD) comparisons of the vehicle accelerations, suspension deflections, and tire dynamic loads between the e-DVA module and the in-wheel motored system in frequency domain are sketched from

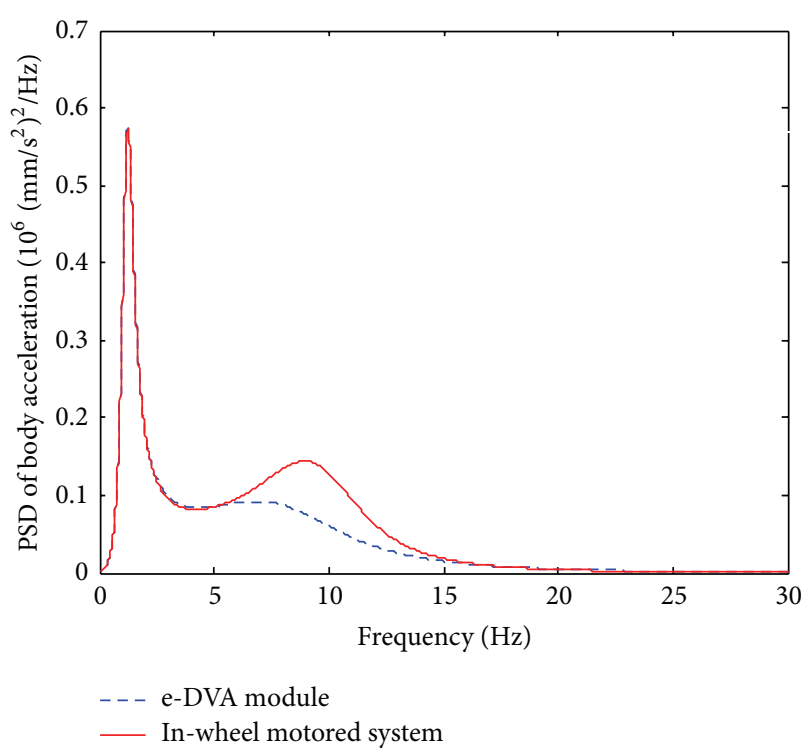

FIGURE 10: PSD comparison of body acceleration.

Figures 10-12. And Figure 13 shows the power spectral density of DVA deflection.

From Figures 10-13, it is shown that, in the human sensitive frequency range of $4-8 \mathrm{~Hz}$, the power spectral density of body acceleration in the e-DVA module decreases significantly comparing with the in-wheel motored system. In the whole frequency range, the second peak is shifted and damped, as well as in the PSD diagrams of suspension deflection and tire dynamic load. It can be explained as follows. As in-wheel motored system is a typical twodegree-of-freedom system, there are two peak values in the frequency domain response, as depicted from Figures 10-12, in which, the first peak value corresponds to the amplitudes of suspension performances at the body natural frequency, while the second peak value corresponds to the amplitudes at the wheel natural frequency. In this paper, transferring the motor mass as the DVA mass from the wheel, the ratio of sprung mass to the unsprung mass is increased, and the wheel natural frequency is reduced. As a result, the second peak value is shifted to the lower frequency. On the other hand, as the motor is transferred from the wheel, the negative effect of heavy unsprung mass is decreased. Further, the DVA owns the function of attenuating the vibration amplitude at the resonance frequency. Consequently, the amplitude is damped at the second resonance frequency. 


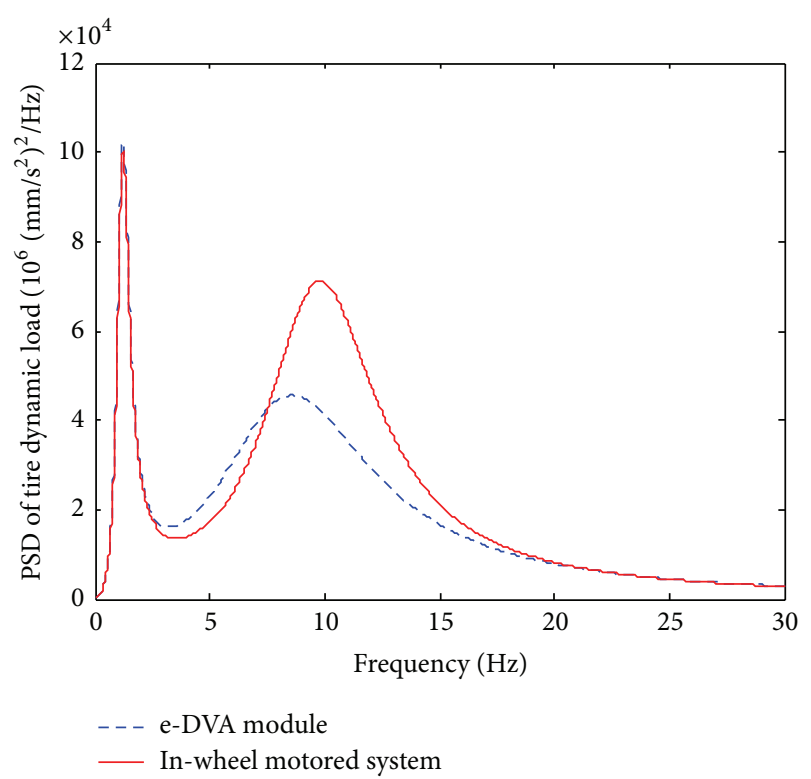

FIGURE 11: PSD comparison of tire dynamic load.

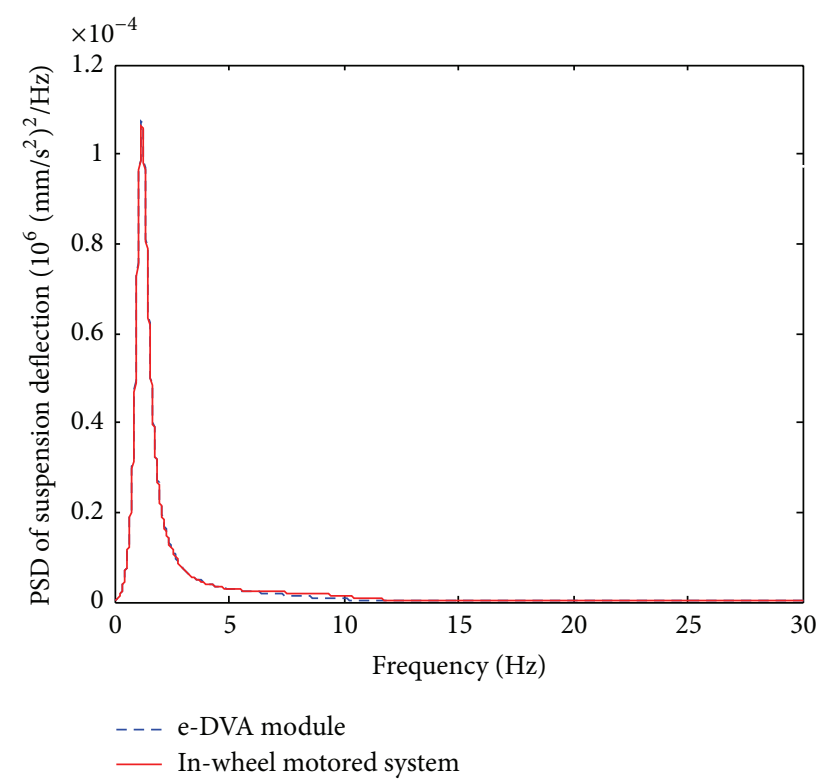

FIGURE 12: PSD comparison of suspension deflection.

4.4.2. Simulation in Time Domain. Also under the excitation of $C$ class road at the speed of $20 \mathrm{~m} / \mathrm{s}$, the body accelerations, suspension deflections, and tire dynamic loads are compared between the e-DVA module and the in-wheel motored system in time domain, as depicted from Figures 14-16. And Figure 17 shows DVA deflection response.

And the RMS values are computed according to the simulation above, as depicted in Table 3.

The suspension performances are evaluated in both frequency and time domains. The second peak value is very important, which presents significant reduction of the suspension performance amplitude in the range near resonance

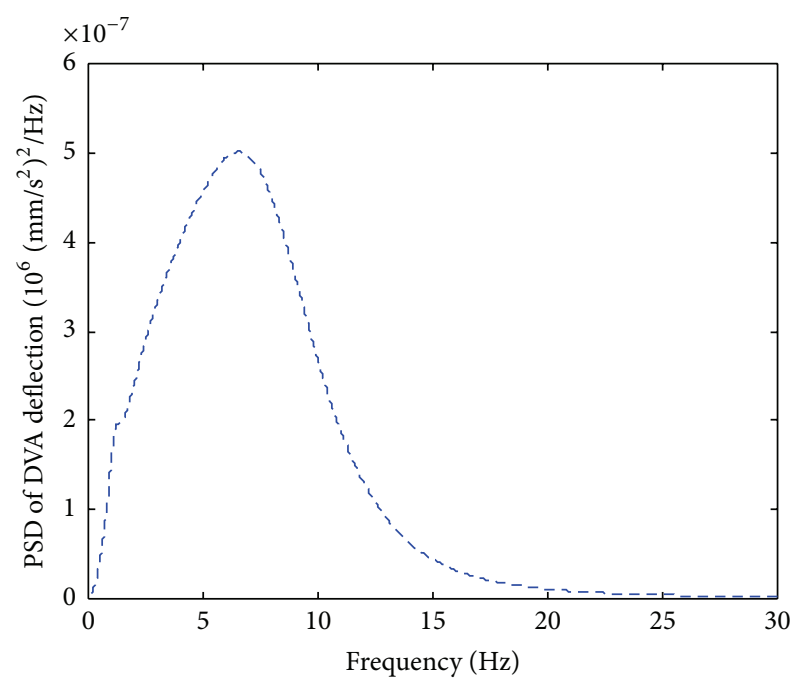

FIGURE 13: PSD of DVA deflection.

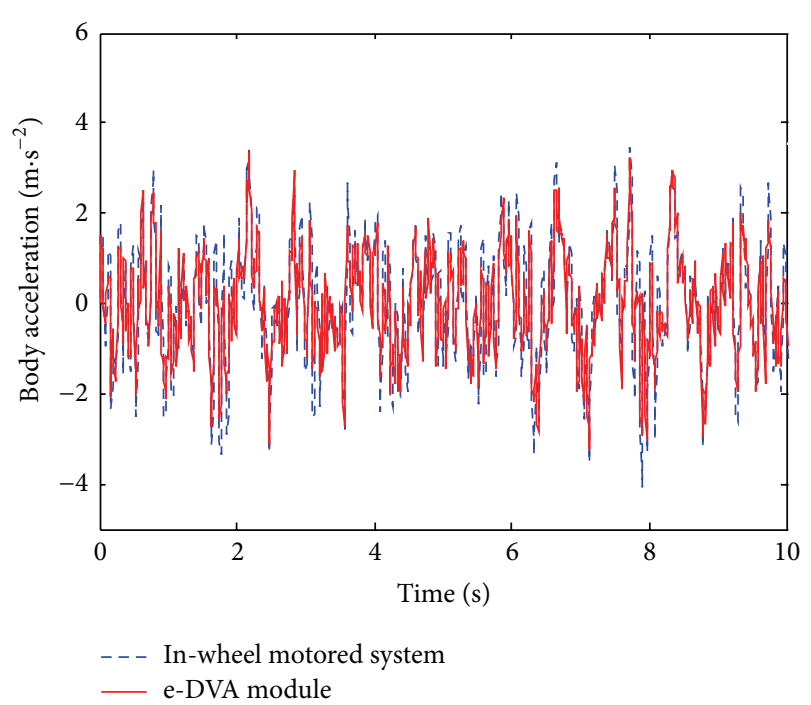

FIgURE 14: Body acceleration variation with time.

frequency. In time domain responses, although the attenuation effect is especially significant in the range near the second resonance frequency, there is no significant difference as the excitation is random including many frequencies. RMS values are utilized to compare the performances numerically, as indicated in Table 3. It is concluded that both the suspension performances of ride comfort and handling stability are improved, as the body acceleration, tire dynamic load, and suspension deflection are reduced $17.88 \%, 15.82 \%$, and $2.70 \%$, respectively.

4.5. Influence Analysis of Speed Ripple. At last, as the transmission ratio is not stable as concluded in Section 2, the influence of speed ripple on wheel speed is analyzed combining with a specific e-DVA module with parameters above. Supposing that the rotational speed of the motor is $5000 \mathrm{r} / \mathrm{min}$, then the wheel speed is $3538^{\circ} / \mathrm{s}$ without any road excitation. 
TABLE 3: Vehicle performance comparisons.

\begin{tabular}{lcc}
\hline & In-wheel motored system & e-DVA system \\
\hline RMS value of body acceleration $/\left(\mathrm{m} \cdot \mathrm{s}^{-2}\right)$ & 1.2920 & 1.1132 \\
RMS value of tire dynamic load $/ \mathrm{N}$ & 866 & 729 \\
RMS value of suspension deflection $/ \mathrm{mm}$ & 11.1 & 10.8 \\
\hline
\end{tabular}

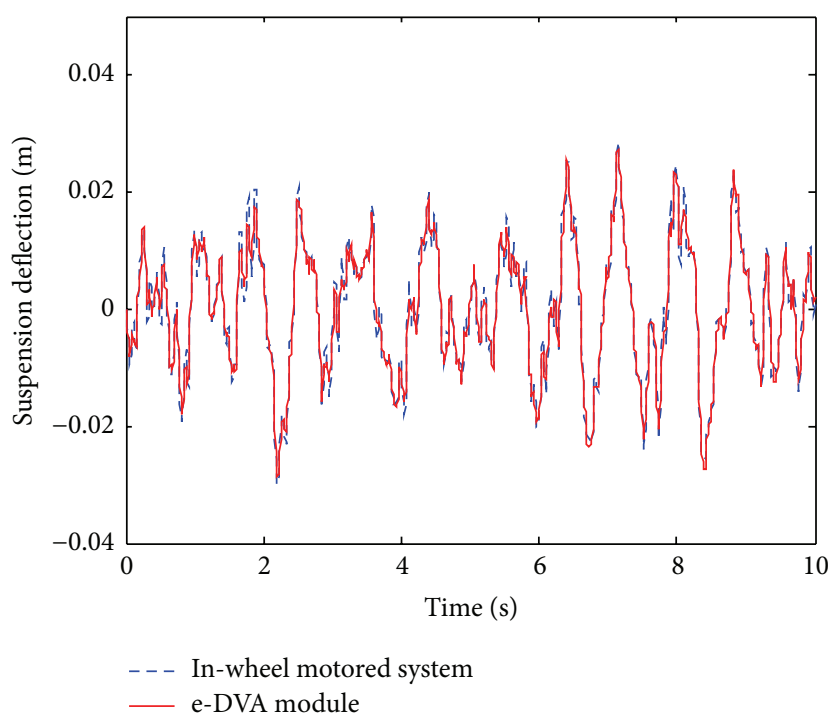

FIgURE 15: Suspension deflection variation with time.

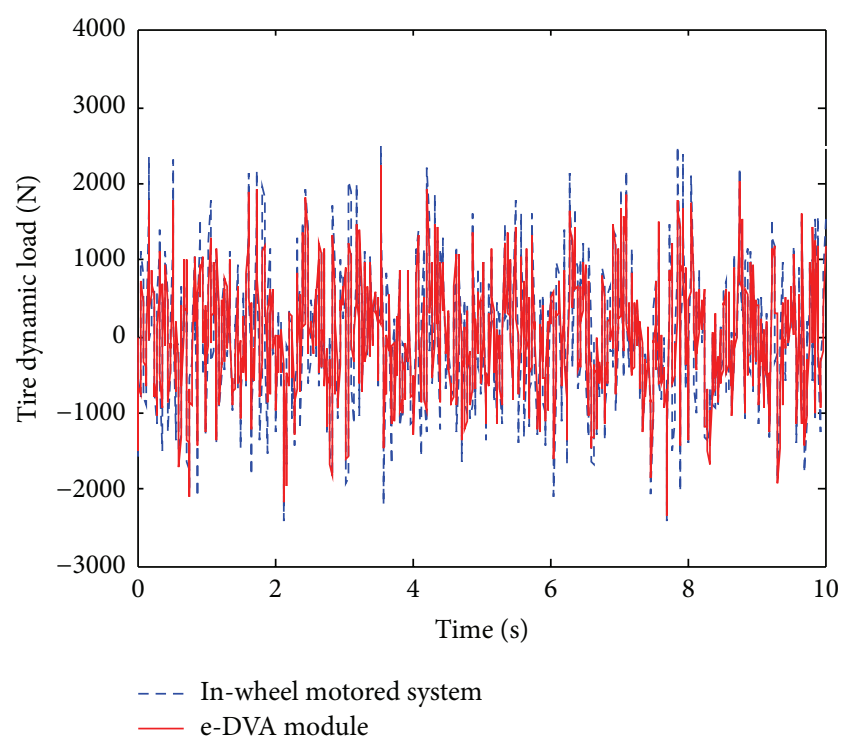

FIgURE 16: Tire dynamic load variation with time.

When the motor performs vertical sinusoidal motion with the amplitude of $6.5 \mathrm{~mm}$ and angular frequency of $2 \pi$ under vibration, the speed ripple is $12.34^{\circ} / \mathrm{s}$ as concluded in ADAMS software. Comparing with wheel speed of $3538^{\circ} / \mathrm{s}$, the speed ripple is much smaller, which has little influence for the common driving and proves the feasibility of application.

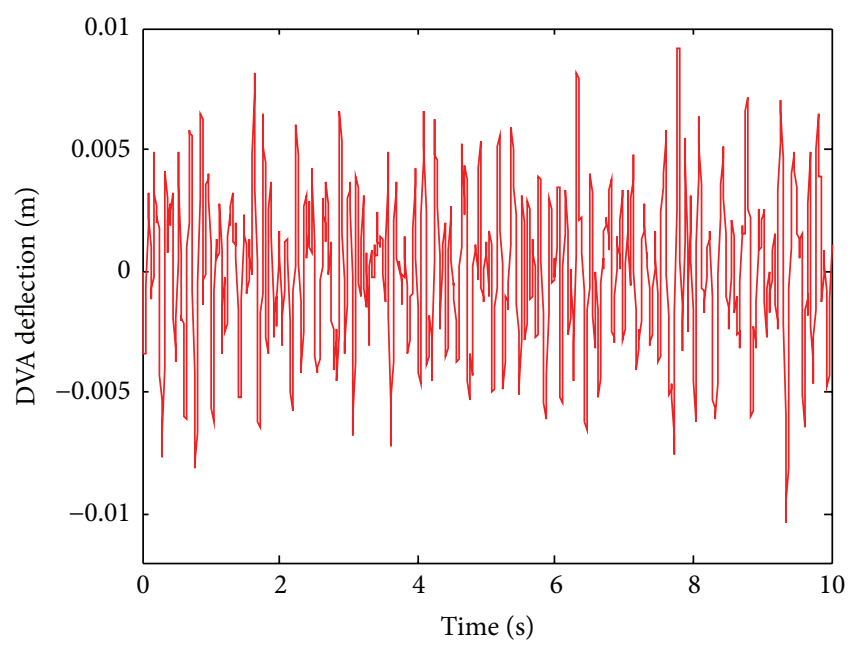

FIgURE 17: Motor deflection variation with time.

\section{Conclusions}

(1) To suppress the negative effects brought by the heavy unsprung mass of the in-wheel motored system, by taking the motor as a dynamic vibration absorber, a novel e-DVA module is proposed in this paper, featuring of the synergy between driving and vibration attenuation. Serving as the crank, the reducer not only transmits the power but also decouples the rigid connection between the motor and the unsprung mass. The e-DVA module presents the advantages of compact structure and low cost.

(2) The stiffness and damping of the e-DVA system are matched and optimized based on weighted $H_{2} / H_{\infty}$ norms of suspension performances. After theory deduction and simulation analysis, comparing with the in-wheel motored system, significant improvements on ride comfort and handling stability are achieved through vertical dynamic analysis in both frequency and time domains. There is little influence of speed ripple on the wheel speed analyzed in ADAMS software, which proves the feasibility of application.

\section{Competing Interests}

The authors declare that there are no competing interests regarding the publication of this paper. 


\section{Acknowledgments}

The authors gratefully acknowledge the support by National Natural Science Foundation of China under Grant no. 51375344

\section{References}

[1] M. Wada, "Research and development of electric vehicles for clean transportation," Journal of Environmental Sciences, vol. 21, no. 6, pp. 745-749, 2009.

[2] Z. Yu, Y. Feng, and L. Xiong, "Review on vehicle dynamics control of distributed drive electric vehicle," Chinese Journal of Mechanical Engineering, vol. 49, no. 8, pp. 105-114, 2013.

[3] D. Hrovat, "Influence of unsprung weight on vehicle ride quality," Journal of Sound and Vibration, vol. 124, no. 3, pp. 497516, 1988.

[4] S. Das, "Design and weight optimization of aluminum alloy wheel," International Journal of Science and Research Publications, vol. 4, pp. 2250-3153, 2014.

[5] Y. Luo and D. Tan, "Lightweight design of an in-wheel motor using the hybrid optimization method," Proceedings of the Institution of Mechanical Engineers, Part D, vol. 227, no. 11, pp. 1590-1602, 2013.

[6] J. F. Eastham, M. J. Balchin, T. Betzer, H. C. Lai, and S. Gair, "Disc motor with reduced unsprung mass for direct EV wheel drive," in Proceedings of the IEEE International Symposium on Industrial Electronics (ISIE '95), pp. 569-573, IEEE, Athens, Ga, USA, July 1995.

[7] W. D. Jones, "Easy ride: bose Corp. uses speaker technology to give cars adaptive suspension," IEEE Spectrum, vol. 42, no. 5, pp. 12-14, 2005.

[8] W. Sun, Z. Zhao, and H. Gao, "Saturated adaptive robust control for active suspension systems," IEEE Transactions on Industrial Electronics, vol. 60, no. 9, pp. 3889-3896, 2013.

[9] C. Xinbo, T. Feng, and X. Lu, "Electric drive system close to wheel with reducer based on single trailing arm suspension," Chinese patent, ZL 201010567793.7, 2011.

[10] X. Chen, J. Yin, C. Gu, F. Tang, and X. Wang, "The research and development of direct wheel drive system with reducer for suppressing unsprung mass negative effects," in Proceedings of the IEEE Transportation Electrification Conference and Expo (ITEC Asia-Pacific '14), pp. 1-6, Beijing, China, September 2014.

[11] S.-C. Huang and K.-A. Lin, "A new design of vibration absorber for periodic excitation," Shock and Vibration, vol. 2014, Article ID 571421, 11 pages, 2014.

[12] X. Zhang and P. Wu, "Study on suspensions with dynamic absorbers," Journal of Jiangsu University (Natural Science Edition), vol. 25, no. 5, pp. 389-392, 2004.

[13] X. Chen, X. Wang, Q. Zhang, and B. Wang, "Simulation and analysis of novel wheel drive system with dynamic damper," Journal of Tongji University(Natural Science), vol. 42, no. 7, pp. 1101-1110, 2014.

[14] B. Agard and S. Bassetto, "Modular design of product families for quality and cost," International Journal of Production Research, vol. 51, no. 6, pp. 1648-1667, 2013.

[15] http://articles.sae.org/4604.

[16] B. Gombert, "eCorner: propulsion by hub motors," Advanced Engineering Materials, vol. 10, no. 5, pp. 503-505, 2008.

[17] D. A. Rade and V. Steffen Jr., "Optimization of dynamic vibration absorbers over a frequency band," Mechanical Systems and Signal Processing, vol. 14, no. 5, pp. 679-690, 2000. 


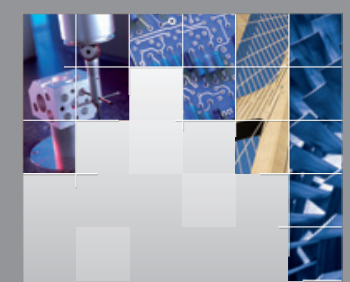

\section{Enfincering}
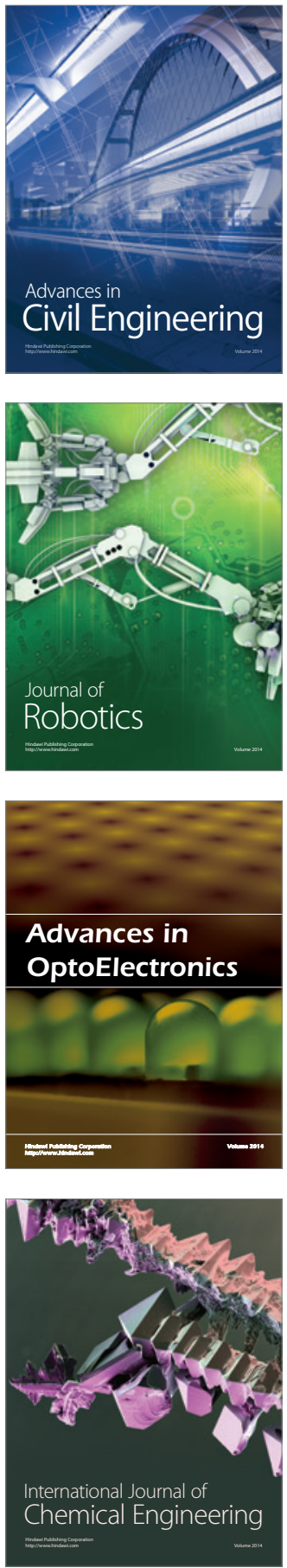

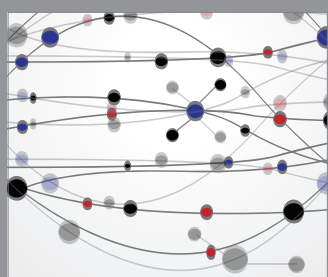

The Scientific World Journal

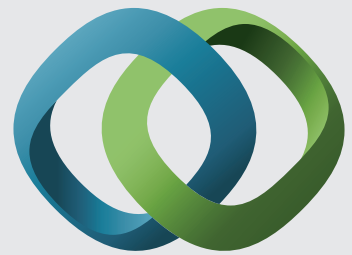

\section{Hindawi}

Submit your manuscripts at

http://www.hindawi.com
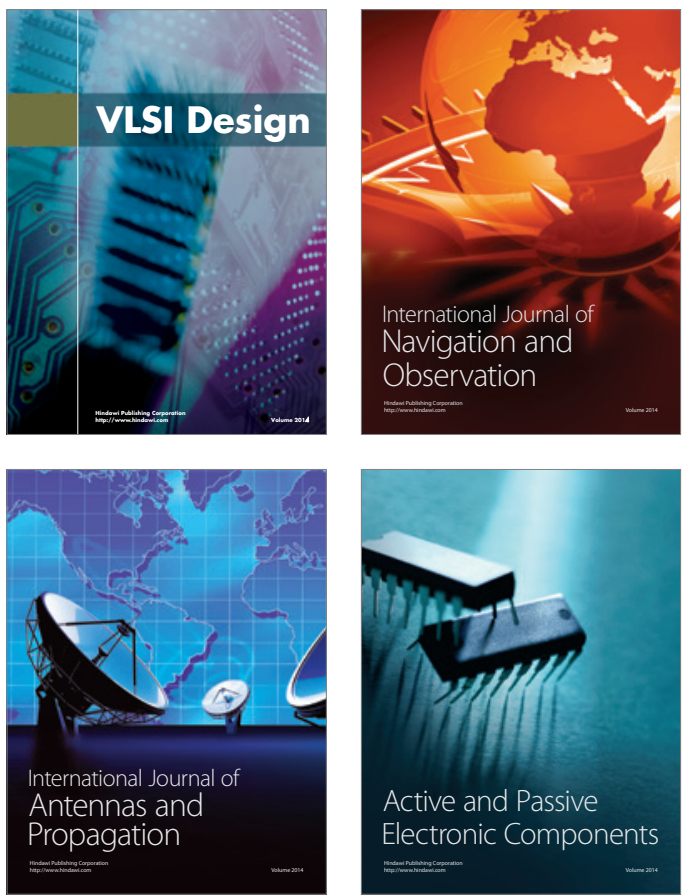
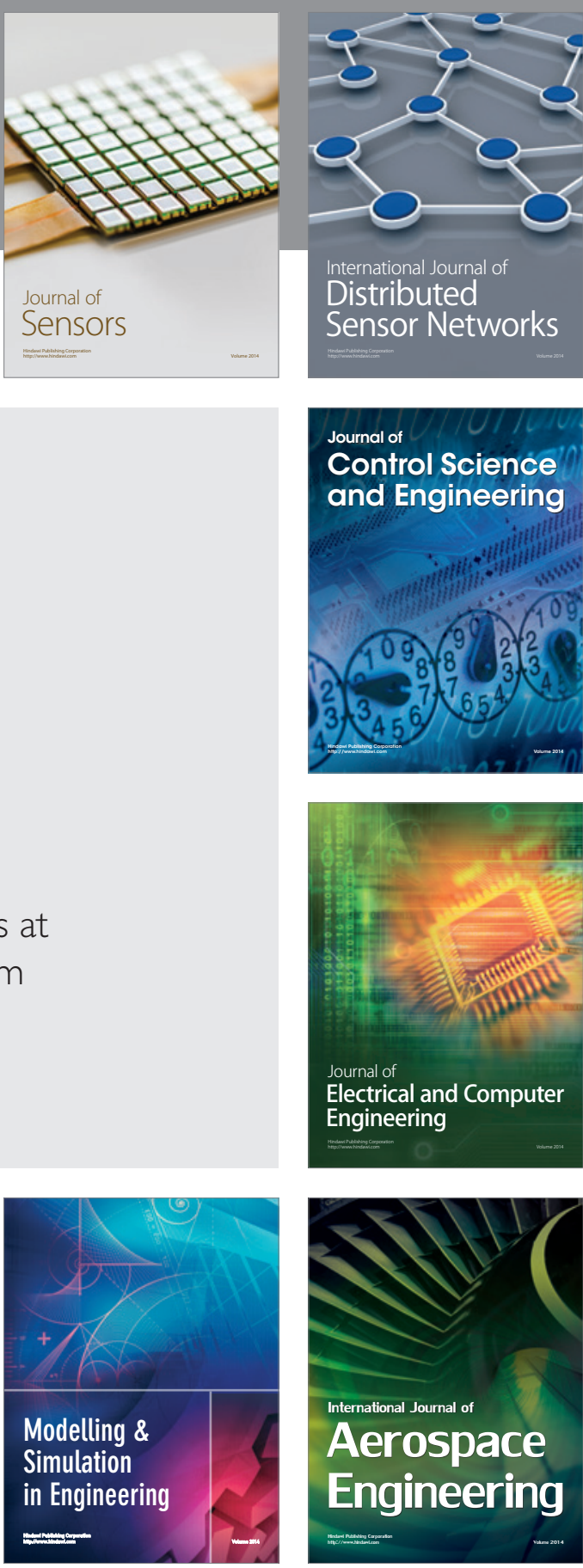

International Journal of

Distributed

Sensor Networks

Journal of

Control Science

and Engineering
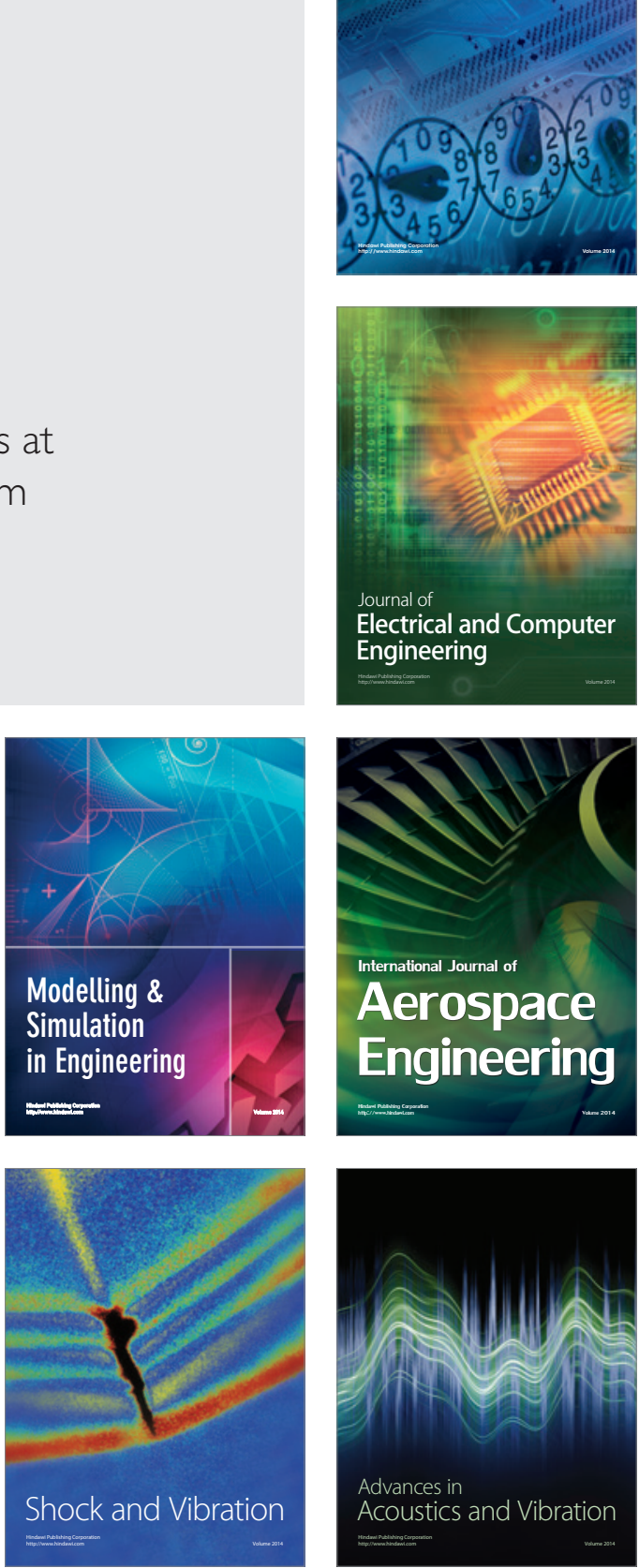